\title{
A major surface glycoprotein of Trypanosoma brucei is expressed transiently during development and can be regulated post-transcriptionally by glycerol or hypoxia
}

\author{
Erik Vassella, ${ }^{1}$ Jan Van Den Abbeele, ${ }^{2}$ Peter Bütikofer, ${ }^{3}$ Christina Kunz Renggli, ${ }^{4}$ André Furger, ${ }^{1}$ \\ Reto Brun, ${ }^{4}$ and Isabel Roditi ${ }^{1,5}$ \\ ${ }^{1}$ Institut für Allgemeine Mikrobiologie, Universität Bern, CH-3012 Bern, Switzerland; ${ }^{2}$ Department of Parasitology, Prince \\ Leopold Institute of Tropical Medicine, B-2000 Antwerp, Belgium; ${ }^{3}$ Institut für Biochemie und Molekularbiologie, CH-3012 \\ Bern, Switzerland; ${ }^{4}$ Swiss Tropical Institute, CH-4002 Basel, Switzerland
}

Differentiation is a means by which unicellular parasites adapt to different environments. In some cases, the developmental program may be modulated by interactions with the host, but the mechanisms are largely unknown. Trypanosoma brucei is transmitted between mammals by tsetse flies. The development of the procyclic form in the tsetse midgut is marked by the synthesis of a new glycoprotein coat, composed of EP and GPEET procyclins, that is important for survival. Here we demonstrate that the composition of the coat changes in response to extracellular signals in vitro and during development in vivo. EP and GPEET are coinduced when differentiation is initiated. Subsequently, EP expression is maintained, whereas GPEET is repressed after 7-9 days. The timepoint at which GPEET is repressed coincides with the appearance of parasites in a new compartment of the fly midgut. In culture, down-regulation of GPEET can be prevented by exogenous glycerol or accelerated by hypoxia. Regulation is post-transcriptional, and is conferred by the

GPEET 3' untranslated region. The same sequence also regulates expression of a reporter gene in the fly. The finding that GPEET is expressed during a defined window during the establishment of infection suggests that it has a specific function in host-parasite interactions rather than a generalized role in shielding underlying membrane molecules.

[Key Words: EP procyclin; GPEET procyclin; tsetse; 3' UTR; differentiation; RNA stability]

Received October 21, 1999; revised version accepted January 18, 2000.

A number of important pathogens, including the protozoan parasite Trypanosoma brucei, which causes human sleeping sickness, rely on insects as vectors for their dissemination. The transmission of $T$. brucei from one mammalian host to the next requires the tsetse fly as an obligate intermediate host. Successful transmission entails the progression of the parasite through a set of distinct life cycle stages and its migration through different insect organs before becoming infective for a new mammalian host.

Two distinct life cycle stages are found in the mammalian bloodstream, a proliferating long slender form and a quiescent short stumpy form that is preadapted for further development in the tsetse fly. When bloodstream forms are ingested by the fly, the stumpy form rapidly

${ }^{5}$ Corresponding author.

E-MAIL isabel.roditi@imb.unibe.ch; FAX 41316314684. differentiates into the procyclic form, which colonizes the posterior midgut (Vickerman et al. 1988) and later crosses (or circumnavigates) the peritrophic matrix and proliferates in the ectoperitrophic space between the matrix and the gut epithelium. In a second phase, known as maturation, the parasite progresses through several more rounds of differentiation as it migrates to the salivary glands (Van Den Abbeele et al. 1999), finally giving rise to the metacyclic form, which is capable of infecting a new mammalian host. Both the strain of trypanosome and the species of tsetse fly have an influence on the transmission rate (Maudlin and Welburn 1994), but the molecular basis is unknown.

The differentiation of bloodstream forms to procyclic forms can be induced efficiently in vitro by a reduction in temperature from $37^{\circ} \mathrm{C}$ to $27^{\circ} \mathrm{C}$ and the addition of cis-aconitate to the culture medium (Brun and Schoenenberger 1981). During the course of differentiation, the parasite's variant surface glycoprotein (VSG) 
coat is replaced by a different coat composed of procyclins. This is accompanied by a switch from glucose to proline as the main energy source. Short stumpy forms, which are able to differentiate synchronously, express procyclin within $2 \mathrm{hr}$ and lose VSG after $4 \mathrm{hr}$ (Ziegelbauer et al. 1990; Rolin et al. 1993; Matthews and Gull 1994; Vassella et al. 1997). The exchange of coat occurs more slowly and asynchronously in long slender forms (Roditi et al. 1989), which first need to reach a particular position in the cell cycle before they are competent to differentiate (Matthews and Gull 1994).

There are two distinct classes of procyclin that are characterized by their internal repeat motifs (Roditi and Clayton 1999). EP procyclins contain up to 30 tandem repeats of glutamic acid-proline, and GPEET procyclin contains 6 pentapeptide repeats (gly-pro-glu-glu-thr). Both types of procyclin are attached to the membrane by the same glycosylphosphatidylinositol anchor (Ferguson et al. 1993; Treumann et al. 1997). The EP procyclins, which are encoded by three distinct genes (EP1-3), can be distinguished by specific residues in their amino termini, differences in the length of the dipeptide repeats and the presence or absence of amino-linked glycosylation sites (summarized in Roditi et al. 1998). GPEET contains no glycosylation sites but, unusual for an extracellular protein, it is extensively phosphorylated on the threonine residues in the pentapeptide repeat (Bütikofer et al. 1997; Mehlert et al. 1999). EP null mutants have been obtained by successive deletion of all three classes of EP (and one of the two copies of GPEET) from the diploid genome (Ruepp et al. 1997). These trypanosomes showed no obvious phenotype in culture, but the frequency with which they established heavy infections in the tsetse fly was 5-10 times lower than the parental line (Ruepp et al. 1997). Attempts to generate GPEET null mutants were not successful, suggesting that the two classes of procyclin are not functionally equivalent.

The stage-specific expression of procyclins is regulated at several levels. Transcription initiation is 6- to 10 -fold higher in the procyclic form than in the bloodstream form (Pays et al. 1990; Vanhamme et al. 1995) and transcription elongation along the polycistronic transcription units is enhanced (Vanhamme et al. 1995). In addition, the $3^{\prime}$ untranslated regions (UTRs) of procyclin mRNAs contain three discrete elements that affect mRNA stability and translation (Hehl et al. 1994; Furger et al. 1997; Hotz et al. 1997; Schürch et al. 1997). Although only the EP1 3' UTR has been analyzed in detail, the same conserved elements are found in the other $3^{\prime}$ UTRs (Hehl et al. 1994; Schürch et al. 1997). The 3' UTR of GPEET is $86 \%$ identical to that of EP1, and includes all three elements, so it is generally assumed that it is coordinately regulated.

Despite the fact that EP and GPEET are both induced when trypanosomes differentiate in vitro (Bütikofer et al. 1999|, the relative amounts of the two classes of procyclin can vary considerably in long-term cultures. Different stocks of $T$. brucei were found to display a whole spectrum of coat phenotypes ranging from $<10 \%$ to $>85 \%$ GPEET (Bütikofer et al. 1997) and even different laboratory cultures of the same stock (427) differed more than fivefold in their relative levels of EP and GPEET (Bütikofer et al. 1997; Ruepp et al. 1997; Treumann et al. 1997; Acosta-Serrano et al. 1999). We also found that GPEET was a minor component of the coat shortly after trypanosomes of the stock STIB 247 were isolated from the tsetse midgut, but became the major species when these cells were maintained in culture for several months (Roditi et al. 1998). In contrast, other trypanosome stocks expressed EP almost exclusively following fly transmission and were unaffected by passage (Bütikofer et al. 1997).

To establish whether these changes in coat composition are an inevitable part of the differentiation program or whether they occur in response to external factors, we have performed a parallel analysis of procyclin expression in trypanosomes differentiating in vivo and in vitro. We show that, in the fly, GPEET expression is a transient event during the establishment of midgut infections. In addition, it can also be modulated in vitro by components of the culture medium or in response to hypoxic conditions. We further demonstrate that developmental regulation of the GPEET mRNA is mediated specifically by the GPEET 3' UTR.

\section{Results \\ Expression of GPEET during synchronous differentiation to procyclic forms}

The pleomorphic strain AnTat 1.1 has the dual advantage that it is fly transmissible, completing the life cycle in a high percentage of infected flies (Le Ray et al. 1977; Delauw et al. 1985; E. Vassella and M. Boshart, in prep.) and that it is possible to obtain a pure population of stumpy forms using defined culture conditions (Vassella and Boshart 1996; Reuner et al. 1997). The kinetics of expression of EP and GPEET procyclins were investigated during synchronous differentiation of stumpy forms in DTM medium by flow cytometry using specific antibodies (see Materials and Methods). EP procyclins rapidly appeared on the cell surface, and after $6 \mathrm{hr}, 84 \%$ of the trypanosomes expressed them at almost maximal levels (Fig. 1A). Likewise, both the unphosphorylated form and the mature phosphorylated form of GPEET were synchronously expressed, but appeared with slower kinetics than the EP procyclins. They could first be detected on the surface 6-12 hr after initiating differentiation and were maximally expressed between 12 and 24 hr.

\section{Culture conditions regulate surface expression} of GPEET

When trypanosomes were further cultured in DTM medium, which was used in the differentiation experiment described above, a slight reduction in the levels of surface EP and GPEET procyclins could be detected 2 days after triggering differentiation; nevertheless, the try- 
A
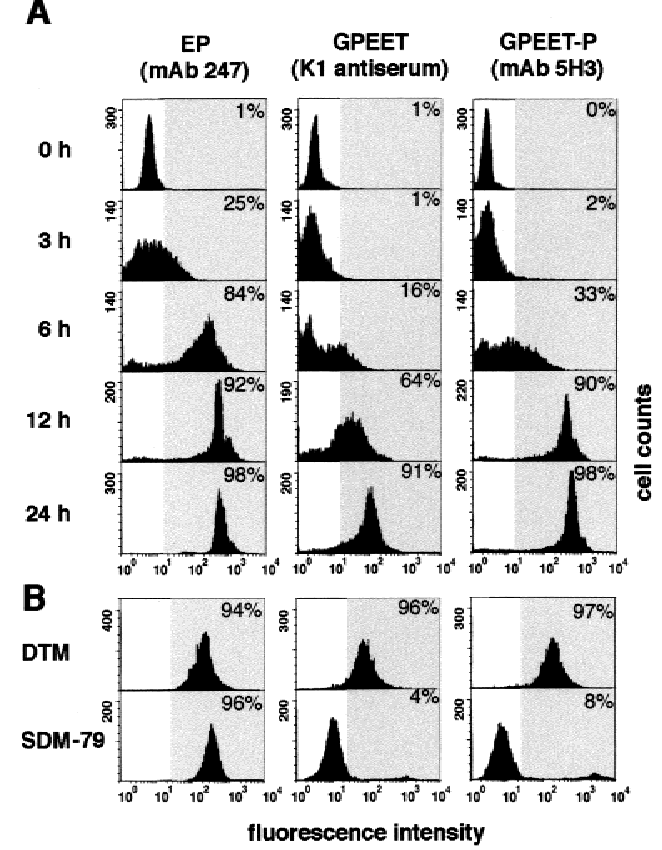

C

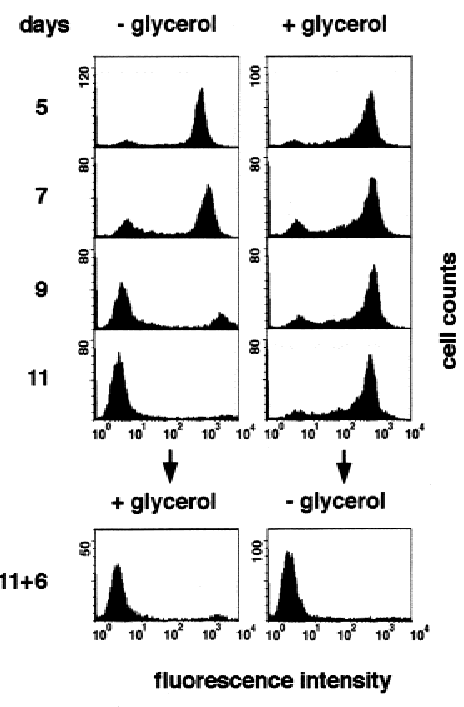

Figure 1. Detection of GPEET and EP procyclins on the surface of trypanosomes by flow cytometry. The percentage of cells showing increased fluorescent staining (within the gray area of the histogram) relative to undifferentiated cells was determined. (A) Appearance of procyclins during synchronous differentiation of stumpy forms into procyclic forms. $(B)$ GPEET surface expression depends on the culture medium. Procyclic forms derived from a stumpy population were cultured in SDM-79 or DTM for 12 days. $(C)$ Constitutive expression of GPEET requires glycerol. Stumpy forms were transferred to normal SDM-79 medium (-glycerol) or SDM-79 supplemented with $10 \mathrm{~mm}$ glycerol (+glycerol) and triggered to differentiate by the addition of cis-aconitate. At day 11 , those trypanosomes that had been previously cultured in the presence of glycerol were cultured for a further 6 days in the absence of glycerol and vice versa. panosomes continued to express high levels of procyclins for at least 8 months (data not shown). In contrast, when stumpy forms were triggered to differentiate in SDM-79 and the resulting procyclic forms were passaged in the same medium, they completely repressed surface expression of GPEET after 2 weeks, but retained EP (Fig. 1B). This suggested that a specific compound in one of the two media might control GPEET expression. The two media were therefore compared and those compounds that differed at least fivefold in their concentrations were selected for further testing. Stumpy forms were triggered to differentiate in SDM-79 or DTM, supplemented with compounds from the other medium, and then analyzed for GPEET expression. By this approach, the active compound could be identified as glycerol, which is present in DTM at a concentration of 10 $\mathrm{mM}$, but is absent from SDM-79. In the absence of glycerol, GPEET was transiently expressed on the surface, but then completely repressed after 9-11 days (Fig. 1C). In contrast, GPEET was constitutively expressed in trypanosomes grown in SDM-79 supplemented with $10 \mathrm{~mm}$ glycerol even after several months in culture.

Repression of GPEET is irreversible and preceded by a transient cell cycle arrest

Procyclic trypanosomes showed a marked increase in the length of the cell body before they repressed GPEET (data not shown). This was accompanied by a transient growth inhibition, usually starting 2-4 days after triggering differentiation and lasting for 4-6 days, before they resumed growth (see Fig. 2). This indicates that early procyclic forms differentiate further to a distinct late procyclic stage that is GPEET negative. In contrast, trypanosomes cultured in DTM or SDM-79 supplemented with $10 \mathrm{~mm}$ glycerol grew with a constant population doubling time of $\sim 20 \mathrm{hr}$ and maintained the same morphology. The percentage of the parasites entering or progressing through the $S$ phase of the cell cycle was determined by incorporation of 5' -bromo-2' deoxyuridine (BrdU) into the DNA during a labeling period of $4 \mathrm{hr}$. Consistent with the results from the growth curve, only $18 \%$ of the cells grown in SDM-79 alone incorporated BrdU after 6 days in culture. In contrast, $86 \%$ of the cells grown in SDM-79 supplemented with glycerol were positive for BrdU.

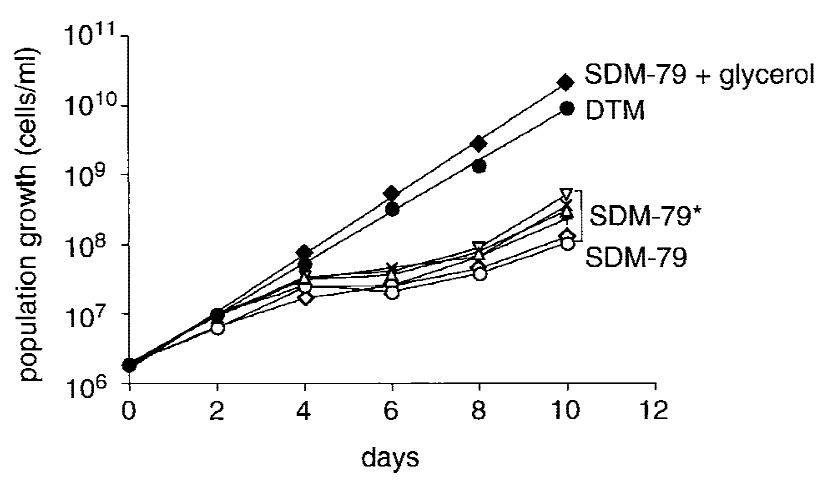

Figure 2. Growth properties of procyclic forms in different medium. Cultures were started from stumpy forms following triggered differentiation (day 0) and diluted at 2-day intervals. The population growth was calculated as cell density multiplied by the cumulative dilution factors. (O) DTM; (O) SDM-79 alone, or ( ) SDM-79 supplemented with $10 \mathrm{~mm}$ glycerol; ( $\nabla)$ 15\% FCS; $(\times) 1.6 \mathrm{~mm}$ glutamate; $(\diamond) 11 \mathrm{~mm}$ glutamine; $(+) 20 \mu \mathrm{M}$ biotin, or $(\triangle) 12 \mu \mathrm{M}$ haemin. SDM-79 supplemented with all compounds from DTM except glycerol is indicated by an asterisk. 
Table 1. Percentage of midgut forms expressing surface EP and/or GPEET procyclins

\begin{tabular}{lrcc}
\hline $\begin{array}{l}\text { Days } \\
\text { p.i. }\end{array}$ & $\begin{array}{c}\text { EP only } \\
(\%)\end{array}$ & $\begin{array}{c}\text { EP + GREET } \\
(\%)\end{array}$ & $\begin{array}{c}\text { GREET only } \\
(\%)\end{array}$ \\
\hline 2 & 4 & 92 & 4 \\
4 & 8 & 88 & 4 \\
7 & 100 & 0 & 0 \\
11 & 100 & 0 & 0 \\
\hline
\end{tabular}

G. morsitans morsitans were infected with short stumpy bloodstream forms of AnTat 1.1.

To investigate whether GPEET repression is a reversible event, trypanosomes that had been kept for 11 days in SDM-79 (and which were negative for GPEET, see Fig. 1C) were transferred to medium supplemented with glycerol and cultured for 6 days. In these cells, glycerol no longer induced reexpression of GPEET on the cell surface. However, cells that were grown in the presence of glycerol for 11 days were still able to repress GPEET at a later time point when glycerol was left out of the medium (Fig. 1C).

Expression of GPEET is a transient event in the establishment of midgut infections in tsetse flies

To investigate whether the repression of GPEET only occurs in vitro, or whether it is an integral part of a developmental process in the life cycle of the parasite, tsetse flies (Glossina morsitans morsitans) were infected with stumpy forms and the percentage of trypanosomes expressing surface EP and/or GPEET was determined during the course of infection. After 2-4 days, the majority of trypanosomes coexpressed both forms of procyclin, but by day 7, all of them expressed high levels of EP but were negative for GPEET (Table 1). GPEET was also not detected in any later life cycle stages (J.V.D. Abbeele, unpubl.). In contrast, EP is not only expressed by midgut forms, but also by epimastigotes in the salivary glands (Vickerman et al. 1988). These results demonstrate that both the induction and subsequent repression of GPEET are events that are programmed to occur in vivo, and with similar kinetics to those observed in vitro.

\section{Glycerol-dependent regulation of GPEET expression is controlled post-transcriptionally}

Procyclin expression is controlled at different levels including transcription initiation and elongation, mRNA stability, and translation (Roditi et al. 1998). To identify the level at which glycerol-dependent regulation of GPEET expression occurred, procyclic forms cultured in the presence or absence of $10 \mathrm{~mm}$ glycerol were compared in the following set of experiments. To investigate whether GPEET was still synthesized, but no longer transported to the surface, in cells that were cultured in the absence of glycerol, immunoblot analysis was performed using the $\mathrm{mAb} 5 \mathrm{H} 3$ (which detects the phos- phorylated form of GPEET). No GPEET protein could be detected in cells cultured in the absence of glycerol (Fig. $3 \mathrm{~A}$ ) indicating that the lack of surface expression was not due to a block in trafficking. The same cells were analyzed for the presence of GPEET mRNA, but again, no hybridization signal was obtained in Northern blots (Fig. 3B). To investigate whether GPEET was regulated by transcription initiation or elongation, nuclear run-on experiments were performed. Similar hybridization signals were obtained when nascent transcripts from GPEETpositive and GPEET-negative cells were hybridized to GPEET DNA (Fig. 3C). The GPEET and EP3 genes form part of a polycistronic transcription unit (Fig. 3D) that

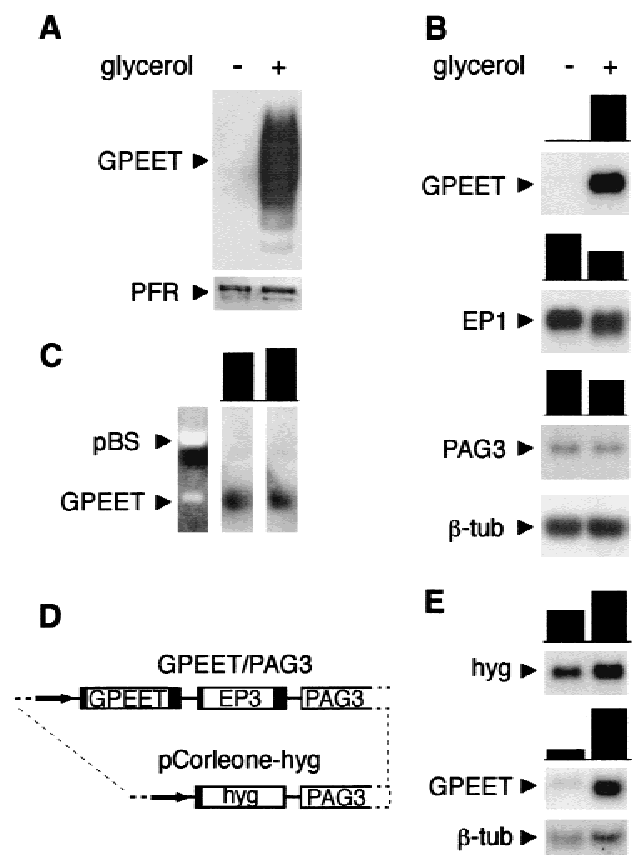

Figure 3. Glycerol-dependent regulation of GPEET expression. (A) Protein expression. Lysates from equal numbers of procyclic forms were analyzed by SDS-PAGE and immunoblotting using the $\mathrm{mAb} 5 \mathrm{H} 3$. An equivalent blot was incubated with an antiserum against the T. brucei paraflagellar rod (PFR) as a control for the sample loading. (B) Steady-state level of mRNA. Total RNA was hybridized with radiolabeled probes from the coding region of GPEET, PAG3, and $\beta$-tubulin or from the 3' UTR of EP1 as indicated in the Materials and Methods section. Hybridization signals were quantified using a PhosphorImager and normalized to $\beta$-tubulin ( $\beta$-tub) mRNA. (C) Nascent RNA expression. Run-on transcripts from isolated nuclei were hybridized to a DNA fragment consisting of the coding region of GPEET released from pBluescript (pBS) (left). The signals were normalized to the amount of RNA hybridized to EP1 (data not shown). (D) Targeting construct to replace the coding region of GPEET and downstream sequences with the hygromycin acetyltransferase gene (hyg). Arrows indicate the promoter, solid boxes the $5^{\prime}$ and 3' UTRs, and broken lines the locus-specific sequences. (E) Northern blot analysis of the trypanosome mutant C-hyg. Total RNA was hybridized with radiolabeled sequences from hyg or probes as indicated in $B$. Procyclic forms of the wild-type $(A, B, C)$ or clone C-hyg $(E)$ were cultured in SDM-79 in the absence $(-)$ or presence $(+)$ of $10 \mathrm{~mm}$ glycerol. 
contains the single copy of the procyclin-associated gene PAG3 (Berberof et al. 1996). The PAGs from the different procyclin loci share common $5^{\prime}$ ends, but they can be distinguished by unique sequences in their $3^{\prime}$ ends (Berberof et al. 1996). Using a probe from the unique region, we could show that the level of PAG3 mRNA was similar in trypanosomes cultured in the presence or absence of glycerol (Fig. 3B). This, in addition to the results obtained from the nuclear run-on experiments, clearly demonstrates that regulation does not occur at the level of transcription. We can therefore conclude that GPEET is regulated post-transcriptionally either at the level of mRNA processing or stability.

The regulatory element is located in the 3' UTR of the GPEET $m R N A$

We first considered a possible role for the $5^{\prime}$ UTR in regulating the steady state levels of GPEET mRNA. If this sequence conferred glycerol-dependent regulation, we would expect that the EP1 mRNA would be regulated the same way, as both transcripts have identical 5' UTRs. However, we consistently observed $30 \%$ higher levels of EP1 mRNA in cells that had repressed GPEET (Fig. 3B; data not shown). We next investigated a derivative of AnTat 1.1 that had been stably transfected with the construct pCorleone-hyg. In this mutant (C-hyg), the coding region of GPEET and downstream sequences were deleted in one of the two GPEET/PAG3 loci and replaced by the hygromycin-resistance gene (hyg) flanked by the 5' UTR of GPEET and the last 19 bp of the 3' UTR of EP3 (Fig. 3D). When procyclic forms of C-hyg were cultured in the absence of glycerol, they did not down-regulate the hyg mRNA (Fig. 3E), suggesting that the control element must be located in the coding region or the $3^{\prime}$ UTR of GPEET.

To distinguish between regulatory elements in the coding region and the $3^{\prime}$ UTR, chloramphenicol acetyltransferase $(C A T)$ cassettes were constructed. Two constructs, pC-CAT/EP1 and pC-CAT/GPEET contained the procyclin promoter from the GPEET/PAG3 (Pro C) locus, whereas a third construct, pA-CAT/EP1, contained the promoter from the EP/PAG1 (Pro A) locus. The CAT gene in the constructs $\mathrm{pA}-\mathrm{CAT} / \mathrm{EP} 1$ and $\mathrm{pC}$ CAT/EP1 was flanked by the $3^{\prime}$ UTR and intergenic region from the EP1 gene and in the construct pC-CAT/ GPEET by the corresponding sequences from the GPEET gene. CAT activity of transiently transfected procyclic forms was expressed as a percentage of that obtained with pA-CAT/EP1. This construct gave rise to similar absolute enzyme activities in trypanosomes cultured in the presence or absence of $10 \mathrm{~mm}$ glycerol (data not shown). The construct pC-CAT/EP1 gave rise to $80 \%$ CAT activity relative to the pA-CAT/EP1 control (Fig. 4), indicating that the GPEET/PAG3 promoter is slightly less active than the EP/PAG1 promoter, at least in an episomal context. The same level of activity was obtained irrespective of whether the cells were cultured in the presence or absence of glycerol, and confirms the

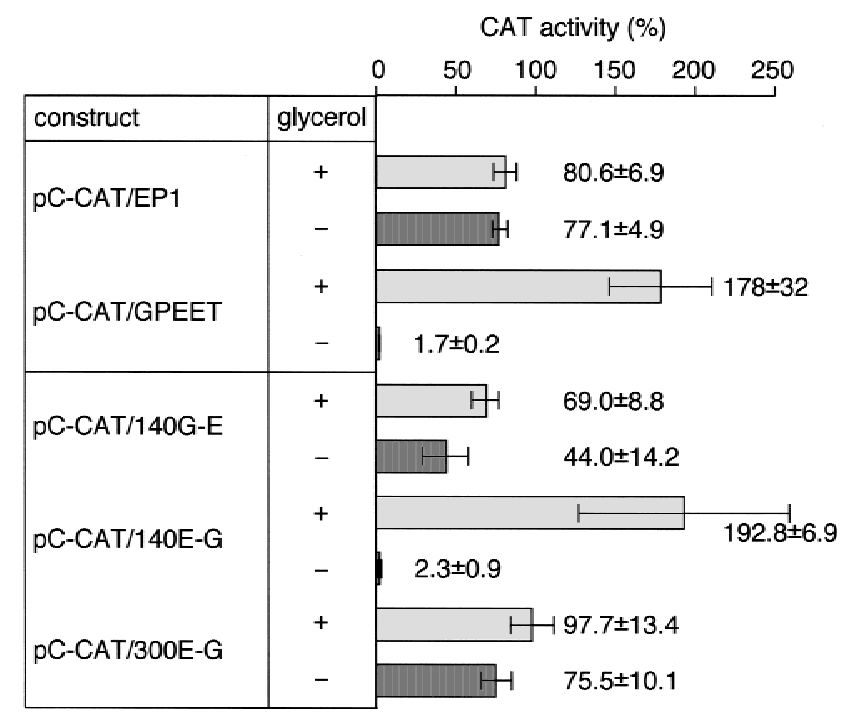

Figure 4. Transient transfections of AnTat 1.1 procyclic forms cultured in SDM-79 in the presence of $10 \mathrm{~mm}$ glycerol $(+)$ or absence of glycerol $(-)$. Values are represented as the means $(n=3) \pm$ S.D. of CAT activities relative to the activity obtained with the construct pA-CAT/EP1.

finding by nuclear run-on analysis that the activity of the GPEET/PAG3 promoter is unaffected. In contrast to the constructs with an EP1 3' UTR, pC-CAT/GPEET produced 100-fold less CAT activity in cells cultured without glycerol than in glycerol-treated cells, demonstrating that expression is controlled by the GPEET 3' UTR. The EP1 and GPEET 3' UTRs did not give equivalent activities in cells cultured in the presence of glycerol. Instead, the GPEET 3' UTR showed a twofold higher activity relative to that of EP1. To further define the regulatory element that controls GPEET expression, three hybrid cassettes were constructed. In the construct pC-CAT/ 140G-E, the first $140 \mathrm{bp}$ of the EP1 3' UTR were replaced by sequences from the GPEET 3' UTR. Conversely, in the construct pC-CAT/140E-G, the first $140 \mathrm{bp}$ of the $3^{\prime}$ UTR were from EP1, whereas the second half of the $3^{\prime}$ UTR and the downstream intergenic region were from GPEET. Transient transfections of trypanosomes with these constructs clearly revealed that the regulatory element must be at least $140 \mathrm{bp}$ downstream of the start of the GPEET 3' UTR: Cells transfected with pC-CAT/ 140G-E showed similar levels of CAT activity in the presence or absence of glycerol, whereas cells transfected with pC-CAT/140E-G showed an 83-fold difference (Fig. 4). In common with pC-CAT/GPEET, the latter construct also showed a twofold higher activity in the presence of glycerol relative to that of pC-CAT/EP1, indicating that the same region must also contain a positive regulatory element. Finally, to distinguish between regulatory elements in the $3^{\prime}$ UTR and intergenic region, we used pC-CAT/300E-G, which contains the entire EP1 3' UTR followed by the GPEET intergenic region. This construct gave rise to similar levels of CAT activity in trypanosomes grown in the presence or absence of glycerol, 
confirming that the regulatory element must be in the GPEET 3' UTR. Because the last 100 nucleotides of the EP1 and GPEET 3' UTR are identical, the regulatory element must lie in the region between nucleotides 140 and 200 of the GPEET 3' UTR.

\section{Regulation of GPEET in the fly}

To investigate at what level GPEET expression is regulated in the fly, we generated a series of stable transformants expressing the Trypanosoma congolense surface protein GARP (glutamic acid/alanine-rich protein) (Bayne et al. 1993; Beecroft et al. 1993). For these experiments, we used bicistronic constructs that were designed to target specific loci and to replace tandemly linked procyclin genes by GARP and an antibiotic resistance gene (shown schematically in Fig. 5A). The deletion of procyclin genes can lead to a reduction in infectivity (Ruepp et al. 1997), but as we have shown recently, this can be overcome by the expression of GARP by procyclic forms of T. brucei (Ruepp et al. 1999). Mutants were derived from $T$. brucei 427, because this stock is particularly easy to culture and to transform, and independent clones can be selected within 2 weeks. When tsetse flies are infected with this stock, it behaves exactly like AnTat 1.1, showing GPEET expression at the beginning of the infection (Fig. 5B; Table 2), but complete repression after $7-14$ days (Fig. 5C; Table 2).

We first analyzed trypanosomes transfected with the construct pGAPRONE-wild type (Furger et al. 1997), in which GARP is fused to the EP1 3' UTR. Two clones, A-GARP/EP1, which had integrated into the EP-PAG1 locus, and C-GARP/EP1, which had integrated into the GPEET/PAG3 locus (shown schematically in Fig. 5A) were selected for further investigation. In both cases, GARP was expressed even 14 days post infection (Fig. 5D,E; Table 2), at which point GPEET was repressed by the majority of cells. The construct pCorleone-GARP, in which GARP is fused to the GPEET 3' UTR (Fig. 5A), was targeted to the GPEET-PAG3 locus. In the resulting clone C-GARP/GPEET, expression of GARP mirrored that of the endogenous GPEET, with most cells expressing both glycoproteins 3 days post infection (Table 2), and neither of them by day 14 (Table 2; Fig. 5F). Trypanosomes isolated from midguts on day 14 were scored for the few cells that still expressed GPEET. Of 22 cells identified, 20 also expressed GARP. Taken together,

Table 2. Percentage of transgenic trypanosomes expressing GPEET and GARP

\begin{tabular}{lcccccccc}
\hline & \multicolumn{2}{c}{$\begin{array}{c}\text { A-GARP/EP1 } \\
(\%)\end{array}$} & & \multicolumn{2}{c}{$\begin{array}{c}\text { C-GARP/EP1 } \\
(\%)\end{array}$} & & \multicolumn{2}{c}{$\begin{array}{c}\text { C-GARP/GPEET } \\
(\%)\end{array}$} \\
\cline { 2 - 3 } \cline { 8 - 9 } $\begin{array}{l}\text { Days } \\
\text { p.i. }\end{array}$ & GARP & GPEET & & GARP & GPEET & & GARP & GPEET \\
\hline 4 & 90 & 55 & & 95 & 97 & & 76 & 78 \\
14 & 80 & 6 & & 85 & 0 & & 0 & 0 \\
\hline
\end{tabular}

G. morsitans morsitans were infected with mutant procyclic forms derived from stock 427.

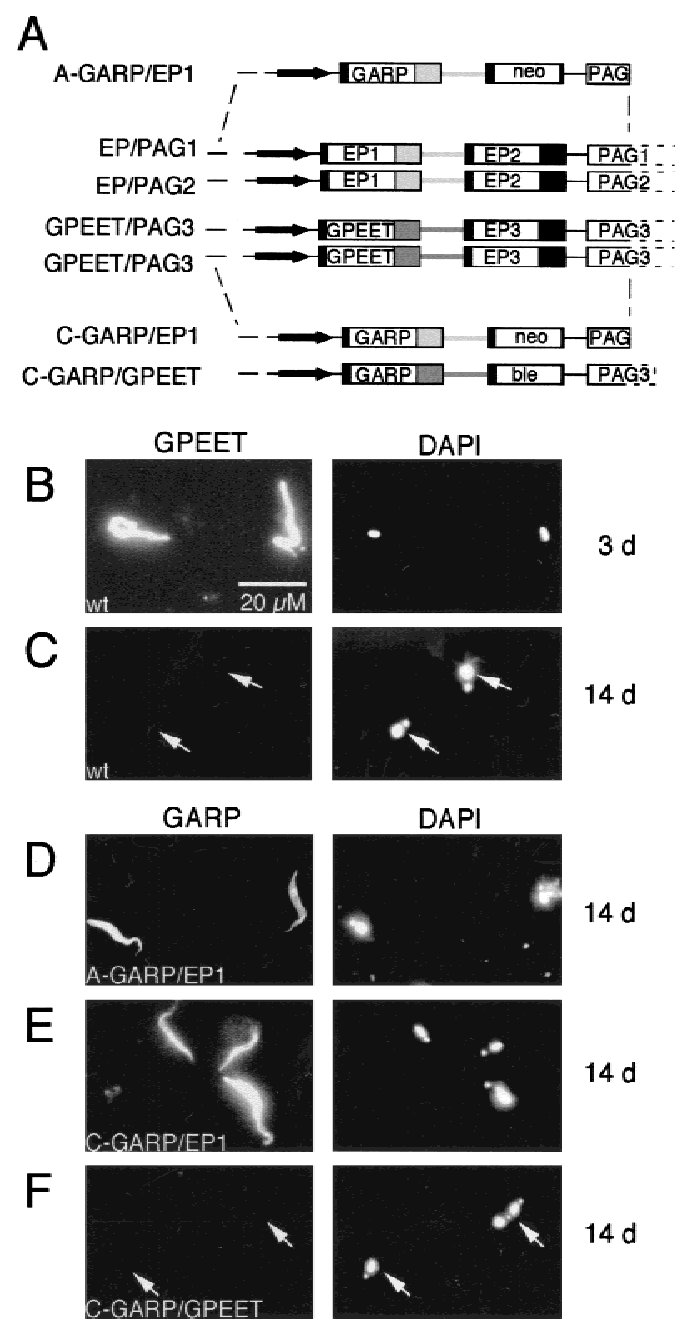

Figure 5. (A) Schematic depiction of the two polymorphic allelic loci $E P / P A G 1$ and EP/PAG2, and the two allelic loci GPEET/PAG3 together with the constructs used to stably transform trypanosomes by homologous recombination. Locus-specific sequences upstream of the promoter region and within the $3^{\prime}$ end of the PAG genes are indicated by broken lines. Arrows indicate the promoter region and solid boxes the $5^{\prime}$ and $3^{\prime}$ UTRs. The 3' UTR and intergenic region from EP1 are indicated by light gray boxes and the corresponding sequences from the GPEET gene are indicated by dark gray boxes. Mutants are named according to the locus that has been targeted (A stands for EP/PAG1, C for GPEET/PAG3) followed by the gene to be expressed and the origin of the $3^{\prime}$ UTR and intergenic sequences. $(B-F)$ Immunofluorescent staining of trypanosomes isolated from the midgut of infected tsetse flies 3 days $(B)$ or 14 days $(C-F)$ after infection with procyclic forms. Wild-type trypanosomes of strain $427(B, C)$ were labeled with anti-GPEET antibodies (K1 antiserum) and the mutant clones A-GARP/EP1 $(D)$, C-GARP/EP1 $(E)$, and C-GARP/GPEET $(F)$ were labeled with a rabbit antiserum against GARP. (Left) The FITC image, (right) the corresponding DAPI image from the same field.

these results demonstrate that it is the 3' UTR, and not the chromosomal context, that determines whether or not GARP and GPEET are coordinately regulated in the fly. 


\section{Repression of GPEET mRNA by hypoxia}

When trypanosomes are ingested by the tsetse fly during a blood meal, might they be transferred to a hypoxic environment? To test whether hypoxic conditions might influence GPEET expression in vitro, trypanosomes grown in medium containing glycerol were transferred to a chamber containing $<1 \%$ oxygen and increased concentrations of carbon dioxide $(>10 \%)$. During the incubation period, trypanosomes showed an increased population doubling time ( $36 \mathrm{hr})$, but no cell death was observed. Under these conditions, glycerol no longer prevented GPEET mRNA repression (Fig. 6). Under the same conditions, the hyg mRNA levels in the mutant $\mathrm{C}$-hyg were unchanged. To further verify that hypoxia did not induce unspecific down-regulation of mRNAs, the same blot was rehybridized with probes from the coding region of $\beta$-tubulin or from the $3^{\prime}$ UTR of EP1. While the levels $\beta$-tubulin mRNA were unaffected, expression of EP1 showed a two- to threefold up-regulation under hypoxic conditions in comparison to normoxic conditions. It remains to be established whether the down-regulation of GPEET and up-regulation of EP1 mRNAs by hypoxia is caused by interference with glycerol catabolism, or whether glycerol and hypoxia control the steady state levels of both mRNAs by independent pathways.

\section{Discussion}

The observation that GPEET is expressed at high levels in some cultures of procyclic form trypanosomes, but at low levels in others, prompted us to investigate its expression in trypanosomes of the fly-transmissible strain AnTat 1.1. During the synchronous differentiation of

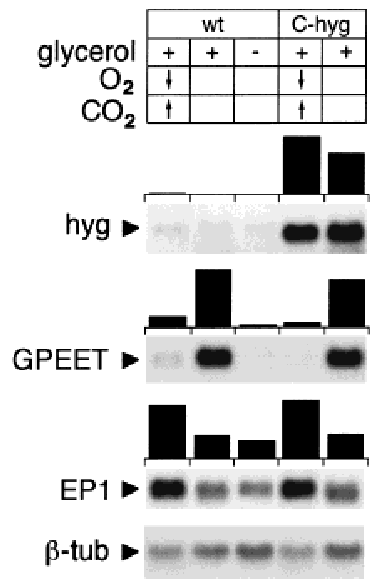

Figure 6. Down-regulation of GPEET mRNA under hypoxic conditions. Total RNA was extracted from procyclic forms cultured under hypoxic conditions $\left(<1 \% \mathrm{O}_{2}\right)$ with increased concentrations of $\mathrm{CO}_{2}(10 \%-20 \%)$, or under atmospheric conditions in the presence $(+)$ or absence $(-)$ of glycerol. Northern blots were hybridized with radioactively labeled probes as indicated in the legend to Fig. 3. Hybridization signals were quantified using a PhosphorImager and normalized to $\beta$-tubulin $(\beta$ tub) mRNA. stumpy bloodstream forms to procyclic forms in vitro, both EP and GPEET were induced and rapidly appeared on the surface, suggesting that the expression of both surface glycoproteins is an important step in the differentiation program.

Procyclic forms cultured in DTM medium grew exponentially and constitutively expressed EP and GPEET. When trypanosomes were transferred to SDM-79 medium, however, they underwent a transient cell cycle arrest 2-4 days after the initiation of differentiation followed by complete repression of GPEET by day 9. This process was irreversible and accompanied by morphological changes. This suggests that an early procyclic form expressing GPEET undergoes a further differentiation step to a late stage that is GPEET negative. Differentiation of slender to stumpy forms in the bloodstream of the host is also preceded by a cell cycle arrest. In this case, however, cell cycle arrest is not transient and trypanosomes need a second signal allowing them to differentiate to the next life-cycle stage, the procyclic form, to resume cell growth (Matthews and Gull 1994; Vassella et al. 1997). Both progression through the cell cycle and GPEET expression were maintained when trypanosomes were cultured in medium containing glycerol, suggesting that these trypanosomes are retained in the early procyclic stage, but can be released from this stage when glycerol is removed. GPEET is also repressed during midgut infection, with slightly faster kinetics than in vitro, indicating that this differentiation step is an integral part of the developmental process in the fly. The time at which GPEET repression occurs correlates with the parasite crossing the peritrophic matrix and colonizing the foregut and the proboscis (Van Den Abbeele et al. 1999). In addition, GPEET-negative trypanosomes most closely resemble anterior midgut mesocyclic trypomastigotes, which are in the ectoperitrophic space. However, it still remains to be seen whether GPEET repression is a cause or a consequence of crossing the peritrophic matrix, or whether it occurs simply because GPEET is no longer needed for survival from this point onward.

It has been reported previously that the monomorphic strain T. brucei 427 predominantly expressed EP shortly after differentiation, but that GPEET was the major surface glycoprotein after long-term passage in SDM-79 (Treumann et al. 1997), and we obtained similar results with the pleomorphic strain STIB 247 (Roditi et al. 1998). At first sight, these observations seem to be contradictory to the results described here, which demonstrate that GPEET is first expressed, but later repressed. It is worth noting, however, that both of these strains had been cultured for 1 month before the first analysis was made, making it likely that the trypanosomes had already repressed GPEET at that time point. To investigate whether GPEET might reappear after long-term culture, we passaged AnTat 1.1 trypanosomes in SDM-79 for 8 months. We confirmed that GPEET was re-expressed after long-term culture, but the expression level was significantly lower than in a population cultured in parallel in medium containing glycerol (E. Vassella, unpubl.). This indicates that re-expression of GPEET can occur 
after prolonged culture in the absence of glycerol, but it is not clear whether the entire population gradually reactivates expression or whether this occurs in a subpopulation that is progressively selected during passage. Although GPEET expression may become independent of glycerol in vitro, as is the case for strain 427 , it is still repressed completely during the course of midgut infections (see Fig. 5). We have shown previously that an EP null mutant (clone Nour 6C) survived very poorly in the fly (Ruepp et al. 1997), suggesting that this class of procyclins either shields the trypanosome from harmful factors, such as digestive enzymes, or is required for positive interactions with tsetse-derived molecules. An alternative explanation, however, might be that these cells would no longer have a procyclin coat once GPEET is repressed, and therefore no longer be viable. This is supported by the observation that the few surviving Nour 6C cells 14 days post infection are positive for GPEET and therefore still coated (E. Vassella, unpubl.), at a point at which wild-type cells are normally GPEET negative.

There is increasing evidence that small molecules play a crucial role in differentiation processes in parasites. Bloodstream form trypanosomes release a soluble factor $<500 \mathrm{Da}$, termed stumpy induction factor (SIF), which accumulates in conditioned medium and which induces differentiation of slender to stumpy forms (Vassella et al. 1997). Differentiation of bloodstream to procyclic forms can also be triggered by a small molecule, cis-aconitate, which, however, is only effective at high concentrations and therefore unlikely to be the physiological trigger. It has also recently been shown for Plasmodium that a mosquito-derived gametocyte-inducing factor is xanthurenic acid (Billker et al. 1998). We now demonstrate that both glycerol and oxygen can regulate the differentiation of procyclic forms in culture. It still remains to be established, however, whether these two signals play a role in fly transmission.

Although GPEET and EP1 are coordinately induced during differentiation of bloodstream to procyclic forms, they are differentially regulated by glycerol, oxygen, and during the establishment of midgut infections. Glycerol-dependent regulation of GPEET is controlled posttranscriptionally. The GPEET 3' UTR was sufficient to confer a glycerol response to a transiently expressed reporter gene. Chimaeras of the EP1 and GPEET 3' UTRs allowed the regulatory element to be mapped to a region between positions 140 and 200 of the GPEET 3' UTR. In stable transformants, the $3^{\prime}$ UTR mediated the developmental regulation of GARP, in concert with GPEET, in the tsetse fly. Hypoxic culture conditions resulted in down-regulation of GPEET, which could be attributed to the coding region and/or 3' UTR. In contrast, EP1 mRNA showed $30 \%$ up-regulation in the absence of glycerol and two- to threefold upregulation under hypoxic conditions. The $3^{\prime}$ UTR of EP1 contains three domains (LI-LIII) that play a role in stage-specific expression (Furger et al. 1997; Hotz et al. 1997; Schürch et al. 1997). LI and LIII both stabilize the mRNA, whereas LII destabilizes it (Furger et al. 1997; Schürch et al. 1997). LI and LIII are conserved in the GPEET 3' UTR $191 \%$ and
95\% identity) and are predicted to form the same secondary structure as in EP1. In contrast, the LII domain is more divergent $(71 \%$ identity to EP1) and is predicted to form a different secondary structure. The 60-bp region encompassing the glycerol-responsive element largely overlaps with LII. This domain may have multiple functions, firstly in the coordinate repression of EP1 and GPEET mRNAs in bloodstream forms, and secondly in their differential response to glycerol. Future experiments should establish whether the same element mediates the response to oxygen and developmental regulation in the fly.

Glycerol and oxygen are both known mediators of gene expression in other organisms. Glycerol controls transcription in a variety of prokaryotes, notably of genes involved in its own catabolic pathway (Smith and Charter 1988; Schweizer 1991; Nilsson et al. 1994) and in chemotaxis (Zhulin et al. 1997). Moreover, it induces the sporulation of vegetative cells of Myxococcus xanthus, thus bypassing the normal development of the intermediate fruiting body (Killeen and Nelson 1988). In most of these cases, glycerol, or a metabolite, is the active compound controlling gene expression, but in other cases, the trigger is the increase in membrane potential induced by glycerol (Zhulin et al. 1997). In procyclic form trypanosomes, glycerol enters the cell by diffusion through the lipid bilayer and is rapidly metabolized for energy production (Ryley 1962; ter Kuile and Opperdoes 1991). At present, it is not clear whether glycerol itself, or a metabolite, regulates GPEET. We have tested a variety of different substrates (glucose, glycerol-3-phosphate, succinate) and inhibitors of glycolysis and oxydative phosphorylation (e.g., rotenone, antimycin A, and salicylhydroxamic acid), but they do not influence GPEET expression. This suggests that regulation does not operate through these pathways.

To the best of our knowledge, our data provide the first example of post-transcriptional regulation by glycerol in eukaryotes. In contrast, there are several examples of eukaryotic transcripts that are regulated post-transcriptionally by hypoxia. These include erythropoietin (McGary et al. 1997) and tyrosine hydroxylase (CzyzykKrzeska et al. 1997), which are regulated by sequences in their 3' UTRs, as well as the more complex example of vascular endothelial growth factor, which requires the cooperation of multiple elements in the coding region and $5^{\prime}$ and $3^{\prime}$ UTRs (Dibbens et al. 1999).

It is well documented that both the trypanosome strain and the tsetse fly species have an influence on the efficiency of cyclical transmission (Le Ray 1989; Moloo et al. 1998) implying that there is cross talk between the parasite and its vector. The finding that environmental signals can translate into differences in coat composition may explain why, in some circumstances, the trypanosome is able to complete its life cycle, whereas in others the infection is confined to the midgut. Apart from GPEET, it is not known whether additional surface molecules are transiently expressed at later stages of the life cycle. These might be specific forms of EP or other, as yet unidentified components of the surface coat. 


\section{Materials and methods}

\section{Trypanosomes}

The pleomorphic strain AnTat 1.1 (Le Ray et al. 1977; Delauw et al. 1985) and the monomorphic strain 427 (Cross and Manning 1973) were used in this study. Bloodstream forms of AnTat 1.1 were cultured on agarose plates as described (Vassella and Boshart 1996; Reuner et al. 1997). A total of $1 \times 10^{5}$ long slender forms were spread per plate and pure populations of stumpy forms were harvested after 4-5 days. Procyclic forms were cultured at $27^{\circ} \mathrm{C}$ in SDM-79 (Brun and Schoenenberger 1979) supplemented with $10 \%$ FBS or in modified DTM medium according to Vassella and Boshart (1996).

\section{Differentiation of bloodstream to procyclic forms and flow cytometry}

Short stumpy bloodstream forms were triggered to differentiate by the addition of $6 \mathrm{~mm}$ cis-aconitate and a drop in temperature to $27^{\circ} \mathrm{C}$ as described (Vassella et al. 1997). Surface expression of procyclin was monitored by flow cytometry using formaldehyde/glutaraldehyde fixed cells (Roditi et al. 1989; Vassella and Boshart 1996). EP and the mature, phosphorylated form of GPEET were detected using the monoclonal antibodies $(\mathrm{mAb})$ TRBP1/247 (Richardson et al. 1988) and 5H3 (Bütikofer et al. 1999), respectively, and the unphosphorylated form of GPEET was detected with the polyclonal rabbit antiserum K1 (Ruepp et al. 1997). TRBP1/247 was used at a dilution of $1: 500,5 \mathrm{H} 3$ at 1:1000, and $\mathrm{K} 1$ at 1:500. FITC-conjugated second antibodies (Dako, Glostrup, Denmark) were used at dilutions of 1:400.

\section{Infection of tsetse flies and isolation of midgut forms}

Pupae of Glossina morsitans morsitans were obtained from the International Livestock Research Institute (Nairobi, Kenya) or the International Atomic Energy Agency (Vienna, Austria). Teneral flies were infected with trypanosomes during the first blood meal after emergence as described (Ruepp et al. 1997). The meal consisted of $2 \times 10^{6}$ short stumpy forms $/ \mathrm{ml}$ defibrinated horse blood (TCS Microbiology, Buckingham, UK) or $2 \times 10^{6}$ procyclic forms/ml SDM-79 supplemented with washed horse red blood cells (Ruepp et al. 1997). Infected flies were fed with three blood meals per week through artificial membranes. Midguts were isolated from infected flies and disrupted by mechanical force in PBS containing $1 \%$ BSA. Large tissue fragments were removed by sedimentation, and the trypanosomes in the supernatant were collected by centrifugation at $1300 \mathrm{~g}$ for $10 \mathrm{~min}$ and spread onto slides.

\section{Immunofluorescence}

Cell smears were air dried and fixed with acetone for $10 \mathrm{~min}$ at $-20^{\circ} \mathrm{C}$. Antibodies that recognize the different forms of procyclins were used at the same dilutions as for flow cytometry (see above). A rabbit polyclonal antiserum raised against a GARPglutathione S-transferase fusion protein (diluted 1:500) was generously provided by D. Jefferies and J.D. Barry (Wellcome Centre of Molecular Parasitology, Glasgow, Scotland). For double immunofluorescence, FITC-conjugated sheep anti-rabbit and TRITC-conjugated goat anti-mouse secondary antibodies (Sigma, St. Louis, MO) were used at dilutions of 1:200. Cells were counterstained with the DNA intercalating dye DAPI. Incorporation of BrdU into the nuclei and kinetoplasts of trypanosomes was performed as described (Vassella and Boshart 1996). To determine the percentage of stained cells, at least 50 trypanosomes were counted per sample.

\section{Cultivation at low $\mathrm{O}_{2}$ and high $\mathrm{CO}_{2}$ concentrations}

Procyclic forms, derived from short stumpy forms and grown for 2 days under atmospheric conditions, were used to initiate cultures in the GENbag system (bioMérieux, Lyon, France). Incubation was performed under hypoxic conditions $\mid<1 \%$ oxygen) and $10 \%-20 \% \mathrm{CO}_{2}$ according to the manufacturer's instructions. Cultures were diluted with fresh medium at 2-day intervals to ensure that the cell density in the GENbag system was between $2 \times 10^{6}$ and $1 \times 10^{7}$ cells $/ \mathrm{ml}$. No cell death could be observed during the incubation period, but after 2-4 days, cells showed a reduced growth rate under hypoxic conditions. Hypoxia was measured by coincubation of the cultures with an indicator strip (bioMérieux). The same pattern of GPEET and EP1 mRNA expression was obtained when trypanosomes were cultured in the BBL GasPak Anaerobic System (Becton Dickinson) under hypoxic conditions with $4 \%-10 \% \mathrm{CO}_{2}$ (data not shown).

\section{Constructs}

Constructs designed to target the GPEET/PAG3 locus (Roditi and Clayton 1999) (formerly known as the Pro C locus; König et al. 1989) contain a SalI/HindIII fragment from clone $\mathrm{pKOH}$ (Ruepp et al. 1997), which includes locus-specific 5' flanking sequences together with the procyclin promoter and the $5^{\prime}$ UTR of the GPEET gene. In addition, all constructs share the same 3' targeting sequences for homologous recombination consisting of the last $19 \mathrm{bp}$ of the EP3 3' UTR, the intergenic region, and the first $950 \mathrm{bp}$ of $P A G 3$. The appropriate fragment was amplified from the genomic clone Pro $\mathrm{C}$ using the primer pair KO1 (containing a BamHI site; Ruepp et al. 1997) and PAGrev (5'GCTCTAGAATTCATGATGGAACGAA-3'; the underlined sequence indicates a $\mathrm{XbaI}$ site introduced to facilitate cloning). The $3^{\prime}$ targeting sequence of $\mathrm{pKOH}$ was excised with BamHI and $\mathrm{XbaI}$ and replaced by the amplification product to generate pCorleone-hyg. This construct was used to generate pCorleone-GARP: In a first cloning step, pCorleone-hyg was linearized with BamHI, the ends were treated with Klenow, and religated to generate a ClaI site. The hyg was released by cleavage with HindIII and ClaI and, in subsequent cloning steps, replaced by 3 fragments (from $5^{\prime}$ to $3^{\prime}$ in pCorleone-GARP) as follows: The first fragment was the GARP coding region, released from pGAPRONE (Furger et al. 1997) by cleavage with HindIII and $B a m H I$. The second fragment consisted of the sequence between the stop codon of GPEET and the start codon of EP3 from the GPEET/PAG3 locus. The appropriate fragment was amplified from the genomic clone Pro $\mathrm{C}$ using the primer pair CPD (5' -AATAGATATCGGATCCAAGCGGATGCAAGCGTGTAA- $\left.3^{\prime}\right)$ and RPU (5'-CAGCGCCGGCAAGCTTGTGAATTTTACTTTTTG-3') and ligated into the HindIII and BamHI sites of pBluescript. The plasmid was linearized with HindIII, the ends were filled with Klenow, and religated to generate a NheI site. This construct was named pBS-intergenic. The insert was released from pBluescript by cleavage with BamHI and NheI and cloned into pCorleone-GARP. The third fragment, located downstream of the other two, consisted of the phleomycin-resistance gene (ble) derived from pBS-phleo (Ruepp et al. 1997). The flanking HindIII and BamHI sites in this construct were first converted into NheI and ClaI sites as described above and together with the other two fragments, cloned into pCorleoneGARP. For stable transformation, all pCorleone constructs were linearized with SalI and XbaI and pGAPRONE-wild type was linearized with KpnI and NotI.

For construction of pC-CAT/GPEET, a HindIII/BamHI fragment containing the GARP coding region was released from 
pCorleone-GARP and replaced by the CAT coding region (Hehl et al. 1994). In a further cloning step, the sequences between $C A T$ and ble in this cassette were released with BamHI and NheI and replaced by the corresponding sequence from the EP/ PAG1 locus, excised from pGAPRONE-wild type (Furger et al. 1997), to generate pC-CAT/EP1. pGAPRONE-wild type was used to generate $\mathrm{pA}-\mathrm{CAT} / \mathrm{EP} 1$ by replacing the coding region of GARP with CAT. CAT constructs containing 3' UTRs that were hybrids between EP1 and GPEET sequences were generated using a common $S s p$ I site 140-bp downstream of the stop codon. A HindIII/SspI fragment containing the CAT gene and the first $140 \mathrm{bp}$ of the EP1 3' UTR was released from pC-CAT/ EP1 and cloned between the HindIII and SspI sites of pC-CAT/ GPEET to generate pC-CAT/140E-G. Likewise, a HindIII/SspI fragment containing the CAT gene and the first $140 \mathrm{bp}$ of the GPEET 3' UTR was released from pC-CAT/GPEET and cloned between the HindIII and SspI sites of pC-CAT/EP1 to generate pC-CAT/140G-E. A hybrid construct containing the EP1 3' UTR and GPEET intergenic region was generated by two sequential amplification steps. The EP1 3' UTR was amplified from pC-CAT/EP1 using a CAT-specific primer $\left(5^{\prime}\right.$-GCGAATTCGGATGAATGGCA-3') and primer 16-(5'-GAAATCTACAGGGCTG-3') from the LIII domain (Furger et al. 1997; Hotz et al. 1997; Schürch et al. 1997), which is identical in EP1 and GPEET. The intergenic region of GPEET was amplified from pBS intergenic using the primer $16+$, which is complementary to 16-, and a Bluescript-specific primer. In a second step, the products of the two PCR reactions were allowed to anneal by hybridization of the complementary 16 mer sequence, amplified using the CAT-specific and Bluescript-specific primers, and cloned between the BamHI and NheI sites of pC-CAT/GPEET to generate pC-CAT/300E-G.

\section{Stable and transient transfection of trypanosomes}

Procyclic forms of the strain 427 were stably transformed as described (Carruthers et al. 1993). After electroporation, cells were transferred to $10 \mathrm{ml}$ of SDM-79 and subsequent dilutions of 1:100 and 1:1000 were performed in SDM-79 containing 25\% conditioned medium (harvested from a stationary phase culture). A total of $1 \mathrm{ml}$ of aliquots were transferred to each well of 24-well plates. After an 18-hr incubation to allow recovery, $1 \mathrm{ml}$ of fresh medium containing $10 \mu \mathrm{g} / \mathrm{ml}$ phleomycin or $100 \mu \mathrm{g} / \mathrm{ml}$ neomycin was added to each well. Independent antibiotic-resistant clonal cell lines were obtained after 10-12 days.

The method used for stable transfection of bloodstream forms of the pleomorphic strain AnTat 1.1 will be described elsewhere (E. Vassella and M. Boshart, in prep.). Transient transfections of AnTat 1.1 procyclic forms were performed as described (Hehl et al. 1994), except that transfected cells were resuspended in SDM-79 supplemented with $30 \%$ conditioned medium.

\section{SDS-PAGE and immunoblotting}

Total cell protein extracts were separated on $12 \%$ polyacrylamide gels and transferred to Immobilon-P (Millipore, Bedford, MA). Detection of procyclins was performed as described (Bütikofer et al. 1999). A polyclonal antibody directed against the paraflagellar rod (PFR) was used at a dilution of 1:1000 (Deflorin et al. 1994).

\section{Nucleic acid analysis}

Northern blot and Southern blot analyses were performed by standard procedures (Sambrook et al. 1989). Multiprime-labeled probes used for hybridization were generated from the coding regions of GPEET, GARP, and hyg, from a fragment from pGAPRONE-wild type (Furger et al. 1997) encompassing the 3' UTR of EP1 and downstream intergenic region up to a $X b a I$ site, from a $P s t$ fragment derived from the $\beta$-tubulin gene or from a EcoRV/SalI fragment from $P A G 3$. Hybridization and washing conditions were $7^{\circ} \mathrm{C}-15^{\circ} \mathrm{C}$ below the calculated melting temperature of the respective probe. Nuclear run-on transcription was performed as described (Berberof et al. 1995). Hybridization and washing was performed at $T_{\mathrm{m}}-7^{\circ} \mathrm{C}$. Hybridization signals were quantified with a PhosphorImager (Molecular Dynamics).

\section{Acknowledgments}

We thank Terry Pearson and Dave Barry for antibodies, Dr Luger, I.A.E.A., Vienna, Austria, for supplying tsetse pupae and Dirk Dobbelaere for constructive comments on the manuscript. This research was supported by grants from the Swiss National Science Foundation, the Helmut Horten Foundation, and the Stanley Thomas Johnson Foundation to I.R. and the Fund for Scientific Research-Flanders (Research Programme G.0358.97) to J.V.D.A.

The publication costs of this article were defrayed in part by payment of page charges. This article must therefore be hereby marked "advertisement" in accordance with 18 USC section 1734 solely to indicate this fact.

\section{References}

Acosta-Serrano, A., R.N. Cole, A. Mehlert, M. Lee, M. Ferguson, and P. Englund. 1999. The procyclin repertoire of Trypanosoma brucei. J. Biol. Chem. 274: 29763-29771.

Bayne, R.A., E.A. Kilbride, F.A. Lainson, L. Tetley, and J.D. Barry. 1993. A major surface antigen of procyclic stage Trypanosoma congolense. Mol. Biochem. Parasitol. 61: 295310.

Beecroft, R.P., I. Roditi, and T.W. Pearson. 1993. Identification and characterization of an acidic major surface glycoprotein from procyclic stage Trypanosoma congolense. Mol. Biochem. Parasitol. 61: 285-294.

Berberof, M., L. Vanhamme, P. Tebabi, A. Pays, D. Jefferies, S. Welburn, and E. Pays. 1995. The 3'-terminal region of the mRNAs for VSG and procyclin can confer stage specificity to gene expression in Trypanosoma brucei. EMBO J. 14: 29252934.

Berberof, M., A. Pays, S. Lips, P. Tebabi, and E. Pays. 1996. Characterization of a transcription terminator of the procyclin PARP A unit of Trypanosoma brucei. Mol. Cell Biol. 16: 914-924.

Billker, O., V. Lindo, M. Panico, A.E. Etienne, T. Paxton, A. Dell, M. Rogers, R.E. Sinden, and H.R. Morris. 1998. Identification of xanthurenic acid as the putative inducer of malaria development in the mosquito. Nature 392: 289-292.

Brun, R. and M. Schoenenberger. 1979. Cultivation and in vitro cloning of procyclic culture forms of Trypanosoma brucei in a semi-defined medium. Acta Trop. 36: 289-292.

- 1981. Stimulating effect of citrate and cis-aconitate on the transformation of Trypanosoma brucei bloodstream forms to procyclic forms in vitro. Z. Parasitenkd. 66: 17-24.

Bütikofer, P., S. Ruepp, M. Boschung, and I. Roditi. 1997. 'GPEET' procyclin is the major surface protein of procyclic culture forms of Trypanosoma brucei brucei strain 427. Biochem. J. 326: 415-423.

Bütikofer, P., E. Vassella, S. Ruepp, M. Boschung, G. Civenni, T. Seebeck, A. Hemphill, N. Mookherjee, T.W. Pearson, and I. 
Roditi. 1999. Phosphorylation of a major GPI-anchored surface protein of Trypanosoma brucei during transport to the plasma membrane. J. Cell Sci. 112: 1785-1795.

Carruthers, V.B., L.H. van der Ploeg, and G.A. Cross. 1993. DNA-mediated transformation of bloodstream form Trypanosoma brucei. Nucleic Acids Res. 21: 2537-2538.

Cross, G.A.M. and J.C. Manning. 1973. Cultivation of Trypanosoma brucei ssp. in semi-defined media. Parasitology 67: 315-331.

Czyzyk-Krzeska, M.F., W.R. Paulding, J.E. Beresh, and S.L. Kroll. 1997. Post-transcriptional regulation of tyrosine hydroxylase gene expression by oxygen in PC12 cells. Kidney Internat. 51: 585-590.

Deflorin, J., M. Rudolf, and T. Seebeck. 1994. The major components of the paraflagellar rod of Trypanosoma brucei are two similar, but distinct proteins which are encoded by two different gene loci. J. Biol. Chem. 269: 28745-28751.

Delauw, M.-F., E. Pays, M. Steinert, D. Aerts, N. Van Meirvenne, and D. Le Ray. 1985. Inactivation and reactivation of a variant-specific antigen gene in cyclically transmitted Trypanosoma brucei. EMBO J. 4: 989-993.

Dibbens, J.A., D.L. Miller, A. Damert, W. Risau, M.A. Vadas, and G.J. Goodall. 1999. Hypoxic regulation of vascular endothelial growth factor mRNA stability requires the cooperation of multiple RNA elements. Mol. Biol. Cell 10: $907-$ 919.

Ferguson, M.A., P. Murray, H. Rutherford, and M.J. McConville. 1993. A simple purification of procyclic acidic repetitive protein and demonstration of a sialylated glycosyl-phosphatidylinositol membrane anchor. Biochem. J. 291: 51-55.

Furger, A., N. Schürch, U. Kurath, and I. Roditi. 1997. Elements in the $3^{\prime}$ untranslated region of procyclin mRNA regulate expression in insect forms of Trypanosoma brucei by modulating RNA stability and translation. Mol. Cell Biol. 17: 4372-4380.

Hehl, A., E. Vassella, R. Braun, and I. Roditi. 1994. A conserved stem-loop structure in the $3^{\prime}$ untranslated region of procyclin mRNAs regulates expression in Trypanosoma brucei. Proc. Natl. Acad. Sci. 91: 370-374.

Hotz, H.R., C. Hartmann, K. Huober, M. Hug, and C. Clayton. 1997. Mechanisms of developmental regulation in Trypanosoma brucei: A polypyrimidine tract in the $3^{\prime}$ untranslated region of a surface protein mRNA affects RNA abundance and translation. Nucleic Acids Res. 25: 3017-3026.

Killeen, K.P. and D.R. Nelson. 1988. Acceleration of starvationand glycerol-induced Myxospore formation by prior heat shock in Myxococcus xanthus. J. Bacteriol. 170: 5200-5207.

König, E., H. Delius, M. Carrington, R.O. Williams, and I. Roditi. 1989. Duplication and transcription of procyclin genes in Trypanosoma brucei. Nucleic Acids Res. 17: 87278739.

Le Ray, D. 1989. Vector susceptibility to African trypanosomes. Ann. Soc. belge Méd. trop. 69: 165-171.

Le Ray, D., J.D. Barry, C. Easton, and K. Vickerman. 1977. First tsetse fly transmission of the "AnTat" serodeme of Trypanosoma brucei. Ann. Soc. belge Méd. trop. 57: 369-381.

Matthews, K.R. and K. Gull. 1994. Evidence for an interplay between cell cycle progression and the initiation of differentiation between life cycle forms of African trypanosomes. J. Cell Biol. 125: 1147-1156.

Maudlin, I. and S.C. Welburn. 1994. Maturation of trypanosome infections in tsetse. Exp. Parasitol. 79: 202-205.

McGary, E.C., I.J. Rondon, and B.S. Beckman. 1997. Post-transcriptional regulation of erythropoietin mRNA stability by erythropoietin mRNA-binding protein. J. Biol. Chem. 272: $8628-8634$.
Mehlert, A., A. Treumann, and M.A. Ferguson. 1999. Trypanosoma brucei GPEET-PARP is phosphorylated on six out of seven threonine residues. Mol. Biochem. Parasitol. 98: 291296.

Moloo, S.K., I.O. Okumu, and N.M. Kuria. 1998. Comparative susceptibility of Glossina longipennis and G. brevipalpis to pathogenic species of Trypanosoma. Med. Vet. Entomol. 12: $211-214$

Nilsson, R.-P., L. Beijer, and B. Rutberg. 1994. The $g 1 p T$ and glpQ genes of the glycerol regulon in Bacillus subtilis. Microbiology 140: 723-730.

Pays, E., H. Coquelet, P. Tebabi, A. Pays, D. Jefferies, M. Steinert, E. Koenig, R.O. Williams, and I. Roditi. 1990. Trypanosoma brucei: Constitutive activity of the VSG and procyclin gene promoters. EMBO J. 9: 3145-3151.

Reuner, B., E. Vassella, B. Yutzy, and M. Boshart. 1997. Cell density triggers slender to stumpy differentiation of Trypanosoma brucei bloodstream forms in culture. Mol. Biochem. Parasitol. 90: 269-280.

Richardson, J.P., R.P. Beecroft, D.L. Tolson, M.K. Liu, and T.W. Pearson. 1988. Procyclin: An unusual immunodominant glycoprotein surface antigen from the procyclic stage of African trypanosomes. Mol. Biochem. Parasitol. 31: 203-216.

Roditi, I. and C. Clayton. 1999. An unambiguous nomenclature for the major surface glycoproteins of the procyclic form of Trypanosoma brucei. Mol. Biochem. Parasitol. 103: 99-100.

Roditi, I., H. Schwarz, T.W. Pearson, R.P. Beecroft, M.K. Liu, J.P. Richardson, H.J. Bühring, J. Pleiss, R. Bülow, R.O. Williams et al. 1989. Procyclin gene expression and loss of the variant surface glycoprotein during differentiation of Trypanosoma brucei. J. Cell. Biol. 108: 737-746.

Roditi, I., A. Furger, S. Ruepp, N. Schürch, and P. Bütikofer. 1998. Unravelling the procyclin coat of Trypanosoma brucei. Mol. Biochem. Parasitol. 91: 117-130.

Rolin, S., P. Paindavoine, J. Hanocq Quertier, F. Hanocq, Y. Claes, D. Le Ray, P. Overath, and E. Pays. 1993. Transient adenylate cyclase activation accompanies differentiation of Trypanosoma brucei from bloodstream to procyclic forms. Mol. Biochem. Parasitol. 61: 115-125.

Ruepp, S., A. Furger, U. Kurath, C.K. Renggli, A. Hemphill, R. Brun, and I. Roditi. 1997. Survival of Trypanosoma brucei in the tsetse fly is enhanced by the expression of specific forms of procyclin. J. Cell Biol. 137: 1369-1379.

Ruepp, S., U. Kurath, C.K. Renggli, R. Brun, and I. Roditi. 1999. Glutamic acid/alanine-rich protein from Trypanosoma congolense is the functional equivalent of ' $\mathrm{EP}^{\prime}$ procyclin from Trypanosoma brucei. Mol. Biochem. Parasitol. 98: 151-156.

Ryley, J.F. 1962. Studies on the metabolism of the protozoa. Biochem. J. 85: 211-223.

Sambrook, J., E.F. Fritsch, and T. Maniatis. 1989. In Molecular cloning: A laboratory manual. Cold Spring Harbor Laboratory Press, Cold Spring Harbor, NY.

Schürch, N., A. Furger, U. Kurath, and I. Roditi. 1997. Contributions of the procyclin 3' untranslated region and coding region to the regulation of expression in bloodstream forms of Trypanosoma brucei. Mol. Biochem. Parasitol. 89: 109121.

Schweizer, H.P. 1991. The agmR gene, an environmentally reponsive gene, complements defective $g l p R$, which encodes the putative activator for glycerol metabolism in Pseudomonas aeruginosa. J. Bacteriol. 173: 6798-6806.

Smith, C.P. and K.F. Charter. 1988. Structure and regulation of controlling sequences for the Streptomyces coelicolor glycerol operon. J. Mol. Biol. 204: 569-580.

ter Kuile, B.H. and F.R. Opperdoes. 1991. Glucose uptake by Trypanosoma brucei. Rate-limiting steps in glycolysis and 
regulation of the glycolytic flux. J. Biol. Chem. 266: 857862.

Treumann, A., N. Zitzmann, A. Hulsmeier, A.R. Prescott, A. Almond, J. Sheehan, and M.A. Ferguson. 1997. Structural characterization of two forms of procyclic acidic repetitive protein expressed by procyclic forms of Trypanosoma brucei. J. Mol. Biol. 269: 529-547.

Van Den Abbeele, J., Y. Claes, D.V. Bockstaele, D.L. Ray, and M. Coosemans. 1999. Trypanosoma brucei spp. development in the tsetse fly: Characterization of the post-mesocyclic stages in the foregut and proboscis. Parasitology 118: 469-478.

Vanhamme, L., M. Berberof, D. Le Ray, and E. Pays. 1995. Stimuli of differentiation regulate RNA elongation in the transcription units for the major stage-specific antigens of Trypanosoma brucei. Nucleic Acids Res. 23: 1862-1869.

Vassella, E. and M. Boshart. 1996. High molecular mass agarose matrix supports growth of bloodstream forms of pleomorphic Trypanosoma brucei strains in axenic culture. Mol. Biochem. Parasitol. 82: 91-105.

Vassella, E., B. Reuner, B. Yutzy, and M. Boshart. 1997. Differentiation of African trypanosomes is controlled by a density sensing mechanism which signals cell cycle arrest via the cAMP pathway. J. Cell Sci. 110: 2661-2671.

Vickerman, K., L. Tetley, K.A.K. Hendig, and C.M.R. Turner. 1988. Biology of African trypanosomes in the tsetse fly. Biol. Cell 64: 109-119.

Zhulin, I.B., E.H. Rowsell, M.S. Johnson, and B.L. Taylor. 1997. Glycerol elicits energy taxis of Escherichia coli and Salmonella typhimurium. J. Bacteriol. 179: 3196-3201.

Ziegelbauer, K., M. Quinten, H. Schwarz, T.W. Pearson, and P. Overath. 1990. Synchronous differentiation of Trypanosoma brucei from bloodstream to procyclic forms in vitro. Eur. J. Biochem. 192: 373-378. 


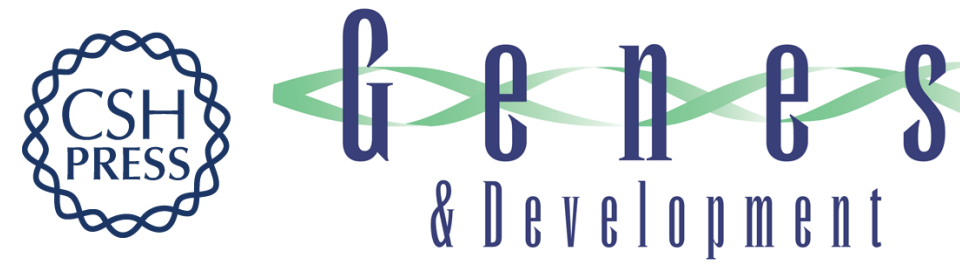

\section{A major surface glycoprotein of Trypanosoma brucei is expressed transiently during development and can be regulated post-transcriptionally by glycerol or hypoxia}

Erik Vassella, Jan Van Den Abbeele, Peter Bütikofer, et al.

Genes Dev. 2000, 14:

Access the most recent version at doi:10.1101/gad.14.5.615

References This article cites 48 articles, 17 of which can be accessed free at: http://genesdev.cshlp.org/content/14/5/615.full.html\#ref-list-1

License

Email Alerting Receive free email alerts when new articles cite this article - sign up in the box at the top Service right corner of the article or click here.

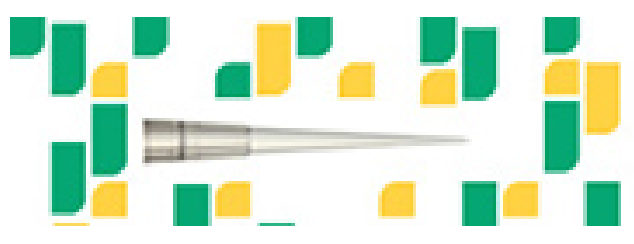

Focused on your science. 